\title{
Application of Block Chain \& Artificial Intelligence in E-commerce supply chain to reduce product counterfeiting $\&$ increase transparency
}

\author{
LAKSHIT JAIN ${ }^{1 *}$, KIRAN KARANDE ${ }^{2}$ \\ ${ }^{1}$ Symbiosis Institute of Operations Management, Nashik, Symbiosis International (Deemed University), Pune, India \\ ${ }^{2}$ Assistant Professor, Symbiosis Institute of Operations Management, Nashik, Symbiosis International (Deemed University), \\ Pune, India
}

\begin{abstract}
Product counterfeiting is a major issue in today's world. These days product counterfeiting leads to around 1.05 lac crore rupees in India per year. As per a report by business standard, if even $50 \%$ of product counterfeiting is stopped with authentication, monitored properly and governed, it can save 50,000 crore rupees per year in India itself, then think about whole world. This research paper is based on the use of block chain along with artificial intelligence to curb the problem of product counterfeiting and maintain the transparency. This research paper also demonstrates the use of blockchain using $\mathrm{R}$ studio as a platform. $\mathrm{R}$ is a programming language mostly for data analytics purposes. So, the implementation of blockchain in $\mathrm{R}$ can give a wide exposure to the data to be analyzed and suitable insights and forecasts can also be made. The research findings give a holistic picture of all the variables that affect the supply chain of E-commerce industry. This study helps the supplier side in curbing their loses and also the consumer side in receiving the genuine product that build trust among both the stakeholders.
\end{abstract}

Article Received: 10 August 2020, Revised: 25 October 2020, Accepted: 18 November 2020

\section{Introduction}

E-commerce has seen amazing growth in recent years. In India, it is forecasted to have a valuation of $\$ 200$ billion in the year 2026 from $\$ 39$ billion in the year 2017. A gateway has opened for consumers to buy products from the sellers all around the world. This growth comes with some challenges, product counterfeiting \& piracy are some among them.

Counterfeiting \& piracy are global problems. From cheap products like toothpastes to expensive products like rolex watches, counterfeiting and piracy are breathing everywhere. Independent ventures, intentionally or accidentally purchase fake innovation or hardware, and endure unforeseen expenses in breakage, business vacation, and unenforceable guarantees. In India, the Government has started steps to address duplicating, theft and pirating. Two significant endeavors stick out. To start with, the legitimate system is genuinely very much evolved in India. Second, the administration has found a way to shield shoppers' health and security from perilous fakes through huge instruction endeavors. Regardless of these activities, an investigation by FICCI shows that fake, theft and pirating rates stay high.

Blockchain was invented by Satoshi Nakamoto in 2008 to create a public transaction ledger for the Bitcoin cryptocurrency. Bitcoin is a decentralized technology which can reduce the tampering of any record to almost none. Blockchain has its application in multiple fields. It can be applied in a supply chain to increase its transparency.

Artificial Intelligence means to develop an intelligence similar to a human into the machines so they can act to some situations without human intervention. Without human intervention there will be lesser tempering to the details which my help to control the product counterfeiting.

The key deliverable from the paper is to understand the concept of blockchain, Artificial Intelligence, Internet of things to reduce the product counterfeiting \& piracy. Also, to prevent consumers from getting a damaged or outdated or expired product by increasing the supply chain visibility and the product details which cannot be tampered by any other party.

The result of this study is expected to give a holistic view of the methodology which can be adapted by the E-commerce companies that can reduce the product counterfeiting $\&$ increase the trust of the customers.

The outline of the paper is follows. Section one provides an introduction of the E-commerce, Blockchain, AI and, how these things combined can be effective in fighting counterfeiting, piracy $\&$ outdated products which are out for sale \&, increase transparency in the supply chain. Section two provides details about the literature review. Section three details about the research 
methodology. Section four discusses about the results obtained through data analysis. This section also provides information about the finding and suggestion. Finally, Section six ends with conclusion.

\section{Literature Review}

An attempt is made to thoroughly review and understand the studies happened earlier on the present research topic. Some of the important references are listed below -

"While the use of network technology to exchange data electronically (e.g. EDIFACT) had existed long before, it was the widespread adoption of the internet for commerce (both B2B and B2C) that heavily impacted supply chains" (al., 2003). "It was not until the commercial potential of the internet was fully realized in the 1990s that the World Wide Web yielded many user-friendly applications with both positive and negative implications for businesses and individuals" (Kambil, 1995). Proponents of the blockchain point out that it enables the creation of a so-called "Internet of Value" (Treiblmaier, 2018). "According to Juniper Research, which conducted a study amongst 369 company founders, executives, managers and IT experts, 76 per cent of respondents believe that the blockchain could be "very useful" or "quite useful" for their company, 35 per cent expect significant internal disruptions and 51 per cent anticipate significant disruptions to their partners or customers" (Holden, 2017). "The whole blockchain market size is estimated to grow from US\$210.2m in 2016 to US $\$ 2,312.5 \mathrm{~m}$ by 2021 , at a compound annual growth rate of 61.5 per cent" (Markets, 2016). "The whole process of validating transactions and adding blocks in a public blockchain is fully distributed such that no single controlling authority exists". (Nakamoto, 2017) (Raval, 2016). "While most popular utilization of blockchain is in cryptocurrency, the Bitcoin, it has other advanced applications and can be used for any trades, contracts, and instalments" (Konstantinos Christidis, 2016) (John Ream, 2016). "Walmart utilizes blockchain to monitor its pork it sources from China and the blockchain records where each bit of meat originated from, prepared, put away and its offer by-date" (Shamout, 2019). "Unilever, Nestle, Tyson and Dole likewise utilize blockchain for comparative purposes. BHP Billiton, the world's biggest mining firm, declared it will utilize blockchain to all the more likely track and record information all through the mining procedure with its sellers, enabling the organization to have more successful correspondence with its partners" (Hackius \& Petersen, 2017). "There are a few production network new businesses, for example, Cloud Logistics who saw a chance to give blockchainempowered inventory network answers for enhanced efficiencies and lesser costs for the enormous store network industry" (Shamout, 2019). "It should then be conceivable to utilize blockchain to execute exchanges and archive trade rapidly, dependably and requiring little to no effort. This blend of the writing proposes that cost-effective logistics could be founded on a cloud logistics display with modern business arrangements in light of small and medium size retail providers and cloud administrations, utilizing blockchain as a halfway arrangement in light of cloud mix. The research will address how blockchain innovation helps supply chain and logistics industry in terms of modern adaptability and change" (Thierry Vanelslander, 2017).

Based on the previous research studies and literature reviewed, it is clear that, there are studies with objective of understanding the application of blockchain \& Artificial Intelligence in supply chain but are very few in regards of product counterfeiting in E-commerce supply chain. Therefore, the study found to be potential new area with the scope of applying blockchain \& $\mathrm{AI}$ in the field of E-commerce.

From extensive investigation of previous research and literature reviewed following conceptual framework was developed for this research. Accordingly, 7 overall stakeholders (i.e. Manufacturer, Distributor, Seller, E-commerce warehouse, E-commerce platform, Buyer \& Logistics) thought to influence the Supply chain are identified. 
Figure 1 Conceptual framework for the study

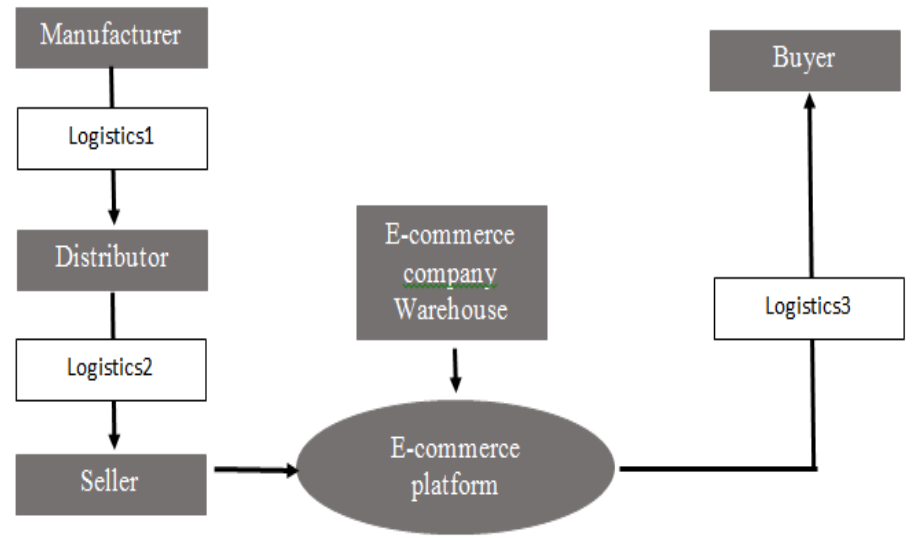

A manufacturer is an individual or an enlisted company which makes completed items from crude materials in an offer to make a benefit. Fundamentally, manufacturers should meet item accreditation prerequisites. This interaction includes execution tests and quality affirmation trial of the products which are being delivered. The certificate bodies underscore on gathering all the pertinent worldwide norms. It's a system to improve consumer protection. A distributor purchases non contending products or product lines, and the resale of the products can likewise be immediate to the client. Distributors frequently have a consented to arrangement with a producer, and this cutoff points them from selling contending products. This arrangement will frequently permit the distributor to portray themselves as a distributor for Product XYZ, however they are not permitted to utilize the XYZ name as a component of their business name. Since they are firmly lined up with the maker, a distributor will likewise regularly offer a scope of administrations to their clients, for example, specialized help, after deal administration, credit, product data, and appraisals. Seller, here is considered to be the retailer listed on the ecommerce platform. A seller will be standing on the other front of the e-commerce for a customer. Buyer is the person who orders the product by using the online platform provided by the ecommerce companies. Logistics in e-commerce is generally handled by the courier companies partnered with the e-commerce companies. These courier companies are responsible for the movement of the orders placed by the buyers by using the e-commerce platforms.

\section{Primary Research -}

\section{Research Methodology}

The main objective of this research is to study the application of block chain \& AI in Ecommerce supply chain to reduce the product counterfeiting and piracy. Therefore, primary research is used to understand the issues related to product counterfeiting and the mindset and requirements of the consumer regarding purchases from the Ecommerce platform.

\section{Analysis -}

a) Type of data -

Both primary data \& secondary data were collected. Primary data were collected with the help of research tool, structured questionnaire, to understand the issued consumer faces and their requirement for supply chain transparency. The objectives of the study have been achieved with help of primary data which were collected from 101 respondents. Secondary data were collected through research reports, books, journals and the latest updates from websites and news. This research is conducted at major cities of India (i.e. Jaipur, Mumbai, Delhi \&, Pune)

\section{b) Data Analysis -}

Data analysis is done using Microsoft Excel. Insights have been generated from the data to analyze the consumer requirements and need of increasing the transparency of the supply chain. 
Figure 2 Insights of the survey
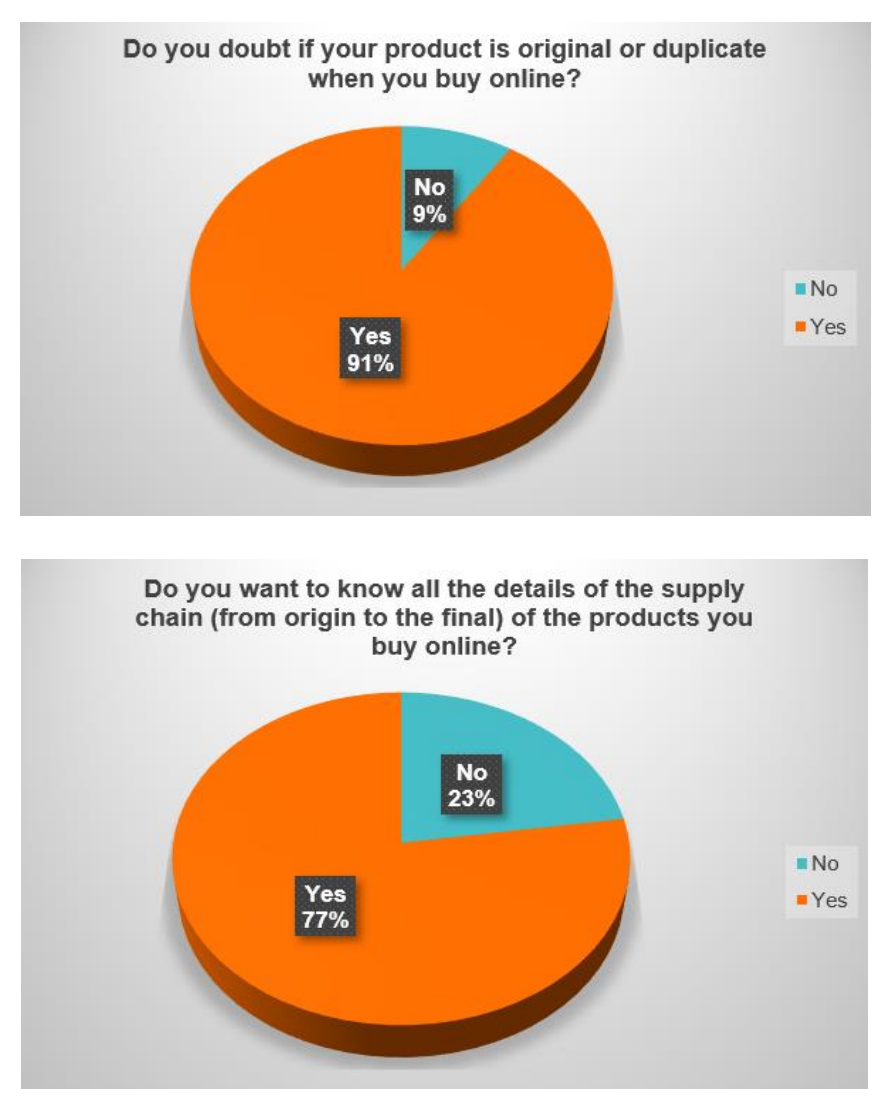

From above insights, it can be concluded that $13 \%$ consumers receive a duplicate product often and $66 \%$ of the consumers have received a counterfeited product at least once. This means there is a scope of improvement in the Ecommerce industry.

\section{Limitations -}

a) Data collection period of 2 weeks is one of the constraints.

b) The study is limited to major cities of India and therefore, the findings of study may vary in other cities.

c) All the findings and observations made in the study are drawn only on the information supplied by the respondents which is based on their preference, ideas and hence there is a chance of bias.

\section{Data generation}

The data can be generated by scanning the UPC (Universal Product Code) and the bar code posted on each of the product packaging. While barcodes were initially evolved to accelerate the sales and exchange measure, they accompany a few

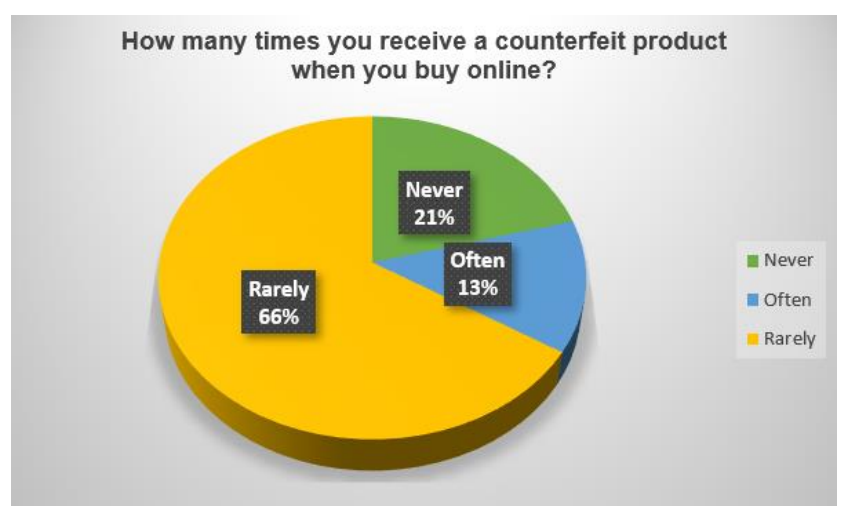

Would you like to track your product with real time on Map?

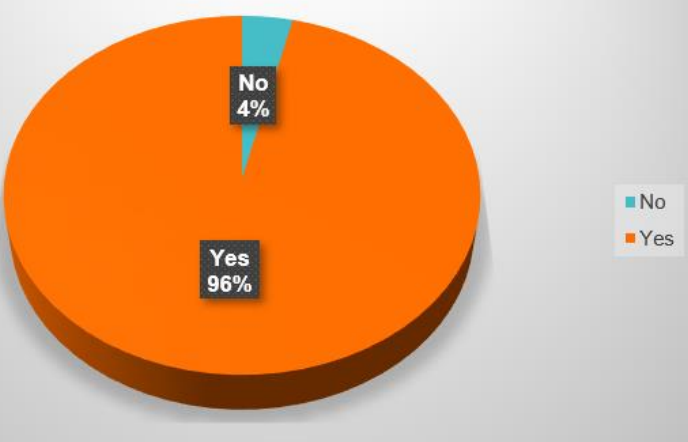

different advantages. Utilizing a barcode to deal with an item's information is considerably more exact than having a sales partner physically enter that information, which is inclined to human blunder. As a result of the speed at which the data is prepared, information about stock levels or sales is accessible right away.

The Universal Product Code is made up of two parts- the bar codes which are readable to machines and the 12 digit number located which is printed under the bar code.

The artificial intelligence enabled devices can scan the bar codes and validates the data stored in it and can match to the data with the blockchain. This Artificial Intelligence enabled device can validate if the product is counterfeited by checking if the blockchain is altered.

In the demonstration shown in the coming parts, each node is considered as the data generated after scanning the bar codes of the product at each vertical of the e-commerce supply chain.

\section{Implementation of Blockchain using $\mathbf{R}$}

Use of Blockchain technology can be a game changer in the field of E-commerce. 
Figure 3 Blockchain Architecture

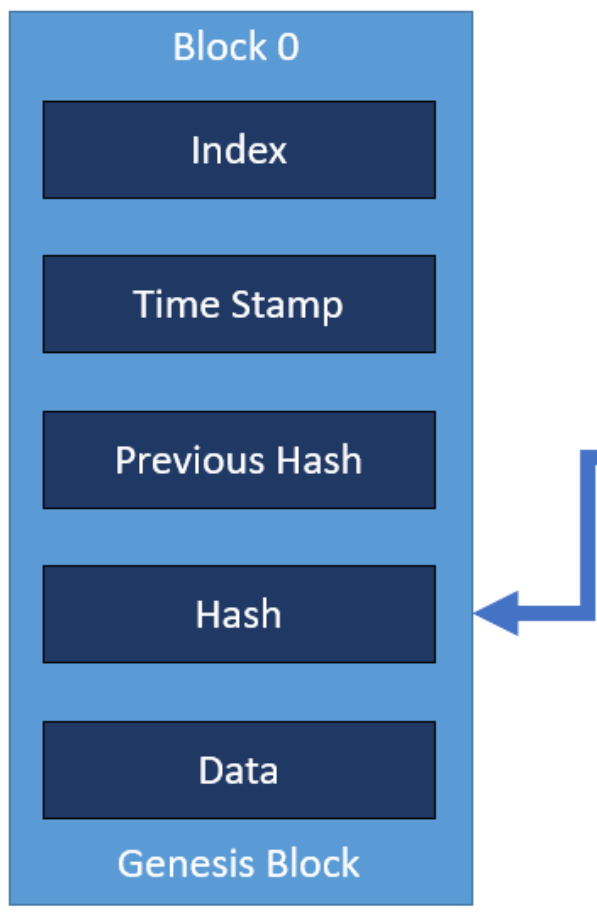

Blockchain Technology enables the data to be stored in a peer to peer network. It is a decentralized network so that no party has full control over the data and it cannot be altered.

The basic blockchain is comprised of different properties:

- Index

- Time Stamp

- Previous Hash

- Hash

- Data

Index represents order of the block. The block with the index 0 is known as genesis block and is the first block in the blockchain. Time Stamp is the indication for the chronological order of blocks. It marks time for every transaction on the blockchain. Time stamp show that happened in the blockchain. Time stamp plays the role of a notary and it is highly credible. Hashing is a type of function which encrypts the data into an alpha numeric form. Hash of a block can be generated by a combination of the data and the hash of a previous block. Hash values are deterministic and respond to the parameters of the given variables of the algorithm. The same sequence cannot be reproduced with a different data set as the input. The resultant hash is irreversible and provides the data a much better security. Data here means the data of the product in the E-commerce supply chain.

Demonstration of the implementation of this blockchain in the E-commerce supply chain has been done using $\mathrm{R}$ programming. To demonstrate the blockchain in supply chain, library "digest" has been used.

\section{library(digest)}

Blocks have been created by using the function "list". Seven blocks representing Manufacturer, Logistics1, Distributor, Logistics2, Seller, Logistics3 \& Buyer are created using R-studio.

Manufacturer is the company or organization that produced the goods those have to be moved in the E-commerce supply chain to reach to the consumer. Logisticsl is the logistics and transportation which connects the manufacturer and the distributor. Distributor oversees the movement of goods from the manufacturer or the supplier to the retailer or the seller in the Ecommerce supply chain. Logistics 2 connects the distributor to the seller or the retailer in the Ecommerce supply chain. Seller is the entity that sells the product on the E-commerce platform and connected to the customer via the same Ecommerce platform. Logistics3 is the transportations and handling that connects the Seller to the buyer.

A block is comprised of "number", "timestamp", "data", "parent hash" \& "hash". Hash has been 
created using the SHA-256 cryptic hash algorithm.

$$
\begin{aligned}
\text { block1 } & <-\operatorname{list}(\text { number }= \\
\text { timestamp } & =\text { " }
\end{aligned}
$$

IST",

data = "Approved by manufacturer", parent_hash $=$ "0")

block1\$hash = $\operatorname{digest}($ block1, "sha256")

$$
\begin{aligned}
& \text { block2 <- list(number }=2, \\
& \text { timestamp }=\text { "2020-09-15 15:22:15 } \\
& \text { IST", } \\
& \begin{array}{l}
\text { data = "Approved by logistics1", } \\
\text { parent_hash = block1\$hash) }
\end{array}
\end{aligned}
$$

block2\$hash $=$ digest $($ block2, "sha256")

block3 <- list (number $=3$, timestamp $=" 2020-09-16 \quad 18: 24: 30$

IST",

data = "Approved by distributor", parent_hash = block2\$hash)

block3\$hash = digest $($ block3, "sha256")

block4 $<-$ list $($ number $=4$,

timestamp $=" 2020-09-17 \quad 16: 24: 00$

IST",

data = "Approved by logistics2", parent_hash = block3\$hash)

block4 $\$$ hash $=\operatorname{digest}($ block4, "sha256")

block5 <- list(number $=5$, timestamp $=" 2020-09-18 \quad 15: 09: 15$

IST", data = "Approved by seller", parent_hash = block4\$hash)

block5\$hash $=$ digest $($ block5, "sha256")

block6 <- list(number $=6$, timestamp $=" 2020-09-19 \quad 14: 07: 30$

IST",

data = "Approved by logistics3", parent_hash $=$ block5\$hash)

block6\$hash $=\operatorname{digest}($ block6, "sha256")

block7 <- list $($ number $=7$, timestamp $=" 2020-09-120 \quad 14: 32: 30$

IST",

data = "Approved by Buyer", parent_hash = block6\$hash)

block7 $\$$ hash $=\operatorname{digest}($ block7, "sha256")
Blockchain has been created by the combination of the blocks. Blockchain is developed using the "list" function in $\mathrm{R}$ programming language. Blocks of every stakeholder created in their respective order in the E-commerce supply chain. blockchain = list (block1, block2, block3, block4, block5, block6, block7)

A function has been created to validate the blockchain. Validate function shows that the blockchain is intact or some data manipulation has been done on to change the data an any of the blocks representing the stakeholders of the Ecommerce supply chain.

validate $\boldsymbol{=}$ function $($ blockchain $)\{$

for (i in 1:length(blockchain)) \{

block = blockchain[[i]]

hash $=$ block\$hash

block\$hash $=$ NULL

hash_expected = digest(block, "sha256")

if (hash != hash_expected) \{ return(FALSE)

\}

\}

if (length(blockchain) $>=2$ ) \{

for (i in 2:length(blockchain)) \{

if (blockchain[[i]]\$parent_hash != blockchain[[i-1]]\$hash) \{ return(FALSE)

\}

\}

\}

return(TRUE)

\}

In the above function, the core idea of the algorithm is that if the data of any block is altered, the respective hash value of the block will also change. As the hash value is the combination of the hash value of previous block, it will affect the previous block and the whole block chain.

Validate(blockchain)

For example, if the data has been changed and the validation function is run again as shown below:

blockchain[[1]]\$data = "xyz"

validate(blockchain)

The result will show "False" as an output. Which means the data in the block chain has been altered. 


\section{a) Conclusion}

\section{Conclusion and Recommendations}

The main objective of this research is to study the application of blockchain and artificial intelligence in the E-commerce industry and its supply chain to improve the transparency to decrease product counterfeiting. IOT devices and use of QR codes can be used to save the data generated, approved and uploaded by the stakeholders in the supply chain over the block chain. The data will be transparent to all the stakeholders so that they can be assured that the data they are approving is original and valid.

According to the survey we have clearly observed the requirement of the transparency in the Ecommerce supply chain. The stakeholders (i.e. Manufacturer, Distributor, Seller, E-commerce warehouse, E-commerce platform, Buyer \& Logistics) holds most of the data in the Ecommerce supply chain. By considering the transactions between these stakeholders as the block on the blockchain, it will be immutable to alter the real data and if someone tries to alter the blockchain all the stakeholders will have the details on which block the data has been altered.

\section{b) Recommendations}

Though the blockchain technology is still niche and unexplored to much extent but it has the potential to break through the limitations and transform the whole e-commerce industry. Blockchain technology has its own concerns such as if the wrong information is put over the blockchain by mistake, you cannot reverse the process. However, a private or consortium blockchain is always an option to these downsides. It has been recommended to make use of blockchain in an efficient way and should be handled with care.

\section{References}

[1] al., L. e. (2003). Strategic Internet application trends in supply chain management. researchgate.net, 32 .

[2] Hackius, N., \& Petersen, M. (2017). Blockchain in logistics and supply chain: Trick or. ECONSTAR, ZBW, 18.

[3] Holden, W. a. (2017). Blockchain Enterprise Survey. Juniper Research, 20.

[4] John Ream, Y. C. (2016). Upgrading blockchains. Delloite.
[5] Kambil, A. (1995). Electronic Commerce: Implications of the Internet for Business Practice and Strategy. Delloite, 20.

[6] Konstantinos Christidis, M. D. (2016). Blockchains and Smart Contracts for the Internet of Things. IEEE.

[7] Markets, M. a. (2016). Blockchain Market by provider, Application. Marketsandmarkets.com.

[8] Nakamoto, S. (2017). Bitcoin:A peer to peer Electronic cash system.

[9] Raval, S. (2016). Decentralized Applications: Harnessing Bitcoin's Blockchain Technology. 15-20.

[10] Shamout, M. (2019). Understanding Blockchain Innovation in. International Journal of Innovative Technology and Exploring Engineering (IJITEE), 7.

[11] Thierry Vanelslander, . S. (2017). Maritime Supply Chains.

[12] Treiblmaier, H. (2018). The impact of the blockchain on the supply chain: a theorybased research framework and a call for action. emerald, 15. 